\title{
Affective Expression among Preterm Infants of Varying Levels of Biological Risk
}

\author{
Gilbert S. Stiefel, James W. Plunkett, and Samuel J. Meisels \\ The University of Michigan
}

\begin{abstract}
This investigation examines the association between risk status and the quality of emotional arousal and regulation among preterm infants in the second year of life. The behovior of 55 preterm infants stratified into three risk groups by severity and chronicity of respiratory illness was recorded during the procedures of the Strange Situation. Measures of emotional responsiveness included temporal and intensity feotures of facial and vocal expressions as well as concurrent activity with toys. Significant relationships between neonatal risk status and these expressive and regulatory features were observed. Infants in the High-Risk group $(N=16)$ differed from healthy Low-Risk infants $(N=23)$ and from those in the Moderate-Risk group $(N=16)$. The High-Risk infants showed a greater sensitivity to distress arousal at low levels of stress and less ability to modulate distress once oroused. High-Risk infonts also demonstrated significantly less adaptive play with toys than the other preterm infants. In short, this study suggests that, when placed under stress, High-Risk preterm infants in their second year of life become more distressed and demonstrate less ability to recover from this distress and effectively re-engage their environment than preterm infonts born at lower risk.
\end{abstract}

\begin{tabular}{|c|c|c|}
\hline affect & $\begin{array}{c}\text { emotional expression } \\
\text { attachment }\end{array}$ & $\begin{array}{l}\text { socioemotional development } \\
\text { prematurity }\end{array}$ \\
\hline
\end{tabular}

This study investigates patterns of affect arousal and regulation in the second year of life among premature infants stratified by severity of respiratory illness. The quality of preterm infant affect regulation has been an important area in the conceptualization of the risk status of prematurity (Emde, Gaensbauer, \& Harmon, 1976; Field \& Fogel, 1982; Lewis \& Michalson, 1983). During the neonatal period preterm infants have been found to have difficulty

This research is based on a dissertation conducted by the first author in partial fulfillment of the requirements for the degree of Doctor of Philosophy in the Rackham School of Graduate Studies at the University of Michigan. It was supported in part by a faculty research award to S.J. Meisels from the Rackham Graduate School. Grateful acknowledgement is made to Sylvia Jones and Leigh Chethik for coding observations, to Patricia Pasick and Dietrich Roloff for their overall assistance in this project, to Ross Thompson for training the coders, to David Cross and Tom Ten Have for methodological consultation, and to the parents and children who participated in the study.

Correspondence and requests for reprints should be addressed to Samuel J. Meisels, Center for Human Growth and Development, The University of Michigan, 300 N. Ingalls, Ann Arbor, MI. 48109 . 
achieving and maintaining adequate state regulation (Als, Lester, Tronick, \& Brazelton, 1982; Duffy, Mowrer, Jensen, \& Als, 1984), with evidence suggesting that these difficulties persist at least into the first months of life. Preterm infants in the first year of life-particularly those who are ill and at high postnatal risk-have been shown to be more difficult to engage, more aversive to social stimuli, and to be more distressed when aroused and less capable of recovering from this distress than full-term infants (Bakeman \& Brown, 1980; DiVitto \& Goldberg, 1979; Field, 1982; Greene, Fox, \& Lewis, 1983). This pattern of vulnerability to stress, disorganization, and difficulty engaging the environment is an important feature of the early transactional difficulties observed between preterm infants and their caregivers (Brachfeld, Goldberg, \& Sloman, 1980; Crnic, Ragozin, Greenberg, Robinson, \& Basham, 1983; Field, 1983; Minde, Whitelaw, Brown, \& Fitzhardinge, 1983).

However, this pattern of affect arousal, regulation, and engagement has principally been observed in the first year of life. The question regarding its persistence beyond the first year remains unresolved. Two studies directly investigating affect expression among healthy, preterm infants suggest an attenuation of differences in expression in the second year. Frodi and Thompson (1985) investigated aspects of facial expression and regulation in groups of 12-month-old healthy full-term and healthy preterm infants, while at 8 and 12 months Brachfeld et al. (1980) studied healthy, full-term infants as well as preterm infants who were either healthy or had experienced only moderate complications at birth. Frodi and Thompson's study coded facial affect expression during the Strange Situation. Brachfeld et al. investigated regulatory and expressive features through observation of the frequency of such affective displays as fretting, crying, or smiling during nonstressful episodes of free play, and infant engagement in toy play. Overall, both studies suggested no significant differences between the preterm and full-term groups at one year in expression of distress or patterns of engagement, thus contributing to the conclusion of a reduction in preterm/full-term differences in affect expression by the end of the first year.

In contrast, Gaensbauer and Harmon (1981) reported that a high-risk group of premature infants studied at one year demonstrated a lower capacity for engagement in activities with toys, as well as significant differences in their range of affect expression when compared with full-term infants. Although it is difficult to fully evaluate these findings because little information about the subjects is available other than their birthweights (all less than $1500 \mathrm{gm}$ ), it is possible that the group differences reported reflect the higher risk status of these infants as compared with the two previous samples. This finding would be consistent with the expectation of persistence of cognitive and affective sequelae among high-risk preterm infants (see Minde et al., 1983). Thus, it follows that affect expression among high-risk preterm infants should be investigated before conclusions regarding the attenuation of sequelae in prematurity in general can be established. 
The major objective of this study is to examine whether preterm infants who differ from one another according to postnatal risk status demonstrate differences in the quality of affect arousal and modulation in the second year of life. Specifically, this study investigates whether high-risk preterm infants in their second year who are placed in a stressful situation become more distressed and demonstrate less ability to recover and effectively re-engage their environment than preterm infants at lower risk.

\section{METHOD}

\section{Subjects}

The sample included 55 premature infants, all of whom were participants in a longitudinal study. The subjects weighed less than $2501 \mathrm{gm}$ at birth and had gestational ages (established by Ballard score [Ballard, Novak, \& Driver, 1979]) of less than 37 weeks. Infants who satisfied the study's selection criteria were identified from case records of consecutive admissions to a large midwestern university hospital between September 1980 and August 1982.

Three groups of infants were defined in terms of chronicity and severity of respiratory illness: High-Risk (HR) $(N=16)$, respiratory illness unresolved within 3 weeks of birth and more than 2 months hospitalization; Moderate-Risk (MR) $(N=16)$, respiratory illness resolved within 3 weeks and 1 to 2 months hospitalization; and Low-Risk (LR) $(N=23)$, no respiratory illness and less than 1 month hospitalization. Subjects in the HK and MR groups had diagnoses of respiratory illnesses that included Respiratory Distress Syndrome, bronchopulmonary dysplasia, Chronic Pulmonary Insufficiency of Prematurity, or Wilson-Mikity syndrome. Mean hospitalization for the HR group was 86 days $(S D=17.9)$, for the MR group 37.7 days $(S D=8.3)$, and for the LR group 10 days $(S D=7.4)$. Ninety-six percent of the families who were asked to participate in the study signed consent forms and were enrolled.

The 3 risk groups were balanced by sex of child, parity, SES, family configuration (i.e., number of parents in home), and maternal education: $55 \%$ of the infants were male, $66 \%$ first-born, $40 \%$ were from low socio-economic status (SES) families (i.e., Classes IV or V of the Hollingshead Four-Factor Index), and $84 \%$ were from two-parent homes. The mean maternal education was 12.6 years $(S D=2.0)$.

As expected, the 3 risk groups differed according to birth weight (BW), gestational age (GA), and birth asphyxia (see Table 1). Since only infants who were Average for Gestational Age were included in the study, the correlation between $\mathrm{BW}$ and GA is very high $(r=.89)$.

The study excluded infants who had one or more of the following disorders: central nervous system, neuromuscular, or sensory disorders; hydrocephalus; intraventricular hemorrhage > Grade II: retrolental fibrophasia; Down's syndrome; cleft palate; severe metabolic disorders; extreme hyperbilirubinemia; or intrauterine growth failure. Infants were also excluded whose mothers had 
TABLE 1

Perinatal Characteristics of Risk Groups: Means and Standard Devications

\begin{tabular}{lccccc}
\hline & \multicolumn{3}{c}{ Risk Groups } \\
\cline { 2 - 4 } & $\begin{array}{c}\text { High } \\
(N=16)\end{array}$ & $\begin{array}{c}\text { Moderate } \\
(N=16)\end{array}$ & $\begin{array}{c}\text { Low } \\
(N=23)\end{array}$ & $\begin{array}{c}\text { Significance } \\
\text { Tests }\end{array}$ & $\begin{array}{c}\text { Significant } \\
\text { Scheffe } \\
\text { Tests }\end{array}$ \\
Perinatal Variables & 1268 & 1600 & 2139 & $F=30.4^{* *}$ & HR $<M R<L R$ \\
\hline Birthweight (gm) & $(502)$ & $(251)$ & $(279)$ & & HR $<M R<L R$ \\
Gestational Age (wks) & 29 & 31.8 & 33.9 & $F=21.25^{\star *}$ & HR \\
& $(2.5)$ & $(1.9)$ & $(1.9)$ & & \\
Asphyxia: & & & & & \\
Apgar $<5$ at 1 min & $43 \%$ & $25 \%$ & $4 \%$ & $\chi^{2}=8.1^{*}$ & (NA) \\
Apgar $<5$ at 5 min & $70 \%$ & $0 \%$ & $0 \%$ & $\chi^{2}=2.65^{*}$ & (NA) \\
\hline
\end{tabular}

Note. Scheffe tests significant at $p<.05$. HR $=$ High-Risk, $M R=$ Moderate Risk, $L R=$ Low Risk.

${ }^{*} p<.02$

$\star * p<.0001$

been addicted to drugs or alcohol during pregnancy or had a severe mental disorder, or were younger than 17 years of age at the time of the child's birth. All infants were living continuously with the same caregivers since hospital discharge.

Approximately half of the subjects were studied at either of 2-time points: 12 months or 18 months Time Post-Hospital Discharge (TPD). This time point was chosen rather than chronological age corrected or uncorrected for prematurity so that all subjects would have comparable opportunities to benefit from the experience of a primary cargiving environment. No significant differences were found in the distributions between these age groups of sex, parity, SES, family configuration, or maternal education.

\section{Procedure}

Infants' emotional responsiveness and toy-play behavior was assessed during their participation in the Ainsworth Strange Situation procedure. The Strange Situation consists of a series of 7 episodes developed to assess infant attachment behaviors under circumstances of increasing and cumulative stress. The social context, which involves the departure and entry or re-entry of the child's primary caregiver (typically, the mother) and an unfamiliar female adult in a scripted sequence, has been described by Ainsworth, Blehar, Waters, and Wall (1978). Sessions were videotaped for later coding.

Facial and vocal modes of emotional expression, as well as concurrent toyplay behavior, were evaluated following procedures described by Thompson (1981), Thompson and Lamb (1984), and Frodi and Thompson (1985). For the vocal expression measure, a number of sensory dimensions including pitch, duration, rhythmicity, and intensity of vocalization were used to classify infant responses along a 12-point continuum. These vocalizations varied along a con- 
tinuum of distress ranging from pleasurable responses to brief whining or fretting, to more continuous and intense vocalizations, such as sobbing and screaming. The facial expression measure focused on organized patterns in the configuration of facial features, particularly in the mouth and eyes/forehead regions. Infant facial features in this procedure were evaluated along a 5-point bipolar scale denoting variation in the quality and intensity of expression. For this scale, ratings 1 and 2 denoted positive expressions, 3 denoted a neutral expression, and 4 and 5 indicated distress expressions that differed in intensity. (A rating of 0 was assigned when an infant's face was not observable for an entire scoring interval and it was impossible to infer the expression from the infant's other behaviors.) Toy play provided a concurrent index of infants' attending to and engagement in other activity. The toy-play scale utilized 10 defined points for assessing the quality of the infant's play with toys and other materiais ranging from no toy play to sustained exploratory play.

\section{Reliability}

Two coders were trained in the scoring procedures on sample videotapes by the author of the scales ( $R$. Thompson). One coder assessed facial expressions and the other coder the vocal expressive modality during 15 scoring intervals. Raters viewed the tapes independently. One of the coders later reviewed the videotapes to rate the toy-play activity. Although this convention may have introduced some nonindependence into the coding, neither of the raters was aware of the purpose of the study or the infants' risk group status. Coding of infant facial and vocal expressions and toy-play behavior was conducted on all 55 infants for whom complete data sets were available. Interrater reliability was assessed through a third rater's independent scoring of all 7 episodes of the Strange Situation for 18 randomly selected subjects. Reliabilities were calculated in two ways. First, the percent of exact agreements between the first author and coder 1 or 2 was established during all scoring intervals on 6 different subjects for each modality. Mean interrater reliability for the Facial Expression Scale was .89 (range $=.87-.92$ ), the mean realiability for the Vocal Expression Scale was .91 (range $=.85-.98$ ), and the mean reliability for the Toy-play Scale was .86 (range $=.82-.92$ ). Second, exact interrater agreement on scores was calculated using Cohen's weighted kappa coefficients (Cohen, 1960, 1968). Weighted kappa values for each of the scales were as follows: Facial Expression $=.82$; Vocal Expression $=.93$; Toy Play $=.87$.

\section{Summary Variables}

Several composite or summary variables were calculated from the facial and vocal affect expression ratings following the procedures first developed by Thompson (1981) and later refined by Frodi and Thompson (1985). There were 3 Intensity/Range measures: Peak Distress Intensity (i.e., highest intensity of distress per episode), Affective Range (i.e., the difference between the highest and lowest intensity rating across all intervals of the episode), and Emo- 
tional Lability (i.e., a function of the total number of transitions between positive and negative ratings across the entire 7 episodes). In addition, there were 4 temporal measures of affect: Distress Latency (i.e., a function of the total number of 15 scoring intervals until the onset of distress during the 2 separation episodes -3 and 5), Rise-Time (i.e., the number of scoring intervals from the initial onset of distress until peak distress intensity is reached during episodes 3 and 5), Initial Distress Recovery, and Final Distress Recovery (i.e., either the initial recovery that is the occurrence of the first nondistressed rating, or final recovery that refers to the occurrence of nondistressed ratings that persist until the end of the episode). Thus, for both the facial and vocal dimensions there were 7 summary affect measures. In addition, the Incidence of Distress was calculated for episodes 3 and 5 (the two primary separation episodes) as the occurrence of any scoring interval in which there was a distress rating during separations. This latter measure was included as a separate description of emotional responsiveness and as an aid in the interpretation of findings from the other composite ratings.

Three summary measures of toy-play behaviors were also calculated. They include: (1) Qualitative Toy Play (derived by counting the number of scoring intervals across each of the 7 episodes that were assigned ratings denoting exploratory play or low-level exploratory play; all types of nonexploratory play activity are excluded from this measure); (2) Quantitative Toy Play (derived in the same manner as the Quantitative Index although ratings of distracted play activity are also included in order to provide a more comprehensive index of toy manipulation by the infant, regardless of quality); and (3) Affective Play Recovery (calculated for the reunion episodes-Episodes 4, 6, and 7-and representing the number of intervals until the infant resumes any type of toy manipulation).

\section{Analysis}

Since Strange Situation episodes varied in length, temporal measures of latency, rise-time, and recovery were each converted to a proportional measure by dividing the number of intervals until distress onset or offset by the total number of intervals in the episode. As suggested by Thompson (1981), the rating scale for the vocal intensity measure was collapsed from 12 to 6 levels to assure that scores reflected interval scaling of the intensity dimension.

Subjects were stratified according to the previously described criteria for analysis. Facial and Vocal Emotional Lability were analyzed in two-way ANOVAs (Risk Group [3] $\times$ Time Post-Discharge [2]). $\Lambda$ three-way repeated measures ANOVA (Risk Group [3] $\times$ Time Post-Discharge [2] $\times$ Episode [7]) was used for the other 15 Affect and Toy-Play measures to rule out Risk $\times$ Time Post Discharge (TPD) interactions, and to evaluate Risk Group main effects and Risk Group $\times$ Episode interactions across the episodes of the Strange Situation. The repeated measures methods accounted for the dependence of Affect and Toy-Play measures taken repeatedly on the same subjects over the several episodes. Homogeneity of error variance assumptions regarding absence of functional relationships between cell means and variances were satisfied via 
square root transformations of intensity and range data for analyses. Contingency table analyses were used for comparisons that included Incidence of Distress.

\section{RESULTS}

The results are examined to rule out the possibility of a Risk Group $\times$ Time Post-Discharge (i.e., age) interaction so that analyses of Risk Group effects could be conducted without stratifying by TPD. The main research question concerning the relationship of Risk to Affect and to Toy Play is addressed by focusing on the 17 main effects for Risk Group, and the 15 Risk Group $\times$ Episode effects. Finally, analyses of the Incidence of Distress are included for descriptive and interpretive purposes.

Risk Group $\times$ Time Post-Discharge Interactions. There were no significant interactions between Risk Group and TPD on any of the measures of Affect and Toy Play. Since the relationships among the Risk Groups did not differ for the 12- and 18-month TPD groups, all subsequent analyses were performed on Risk Groups unstratified by age.

Affect and Toy-Play Measures: Main Effects. There were no significant differences among the Risk Groups in their overall levels of facial or vocal reactivity on Peak Distress Intensity, Affective Range, or Emotional Lability. However, as shown in Table 2, the concurrent measures of Toy Play indicated

TABLE 2

Toy Play Measures: Mean Group Scores

\begin{tabular}{lrrr}
\hline & & \multicolumn{2}{c}{ Risk Group } \\
\cline { 2 - 4 } & $\begin{array}{c}\text { High } \\
(N=16)\end{array}$ & $\begin{array}{c}\text { Moderate } \\
(N=17)\end{array}$ & $\begin{array}{c}\text { Low } \\
(N=22)\end{array}$ \\
\hline Measure & & & \\
Qualitative Index of Toy Ploy & & & 7.17 \\
Episodes & 4.90 & 8.01 & 5.03 \\
1 & 4.19 & 5.26 & 5.75 \\
2 & 2.06 & 6.09 & 7.28 \\
3 & 5.75 & 6.30 & 2.59 \\
4 & 1.69 & 1.82 & 3.85 \\
5 & 3.35 & 3.13 & 5.97 \\
6 & 5.44 & 5.44 & \\
7 & & & 10.09 \\
Quantitative Index of Toy Play & & & 9.85 \\
Episodes & 9.88 & 10.79 & 8.23 \\
1 & 8.75 & 9.78 & 10.01 \\
2 & 4.69 & 8.86 & 5.14 \\
3 & 7.00 & 8.75 & 5.17 \\
4 & 2.94 & 2.93 & 7.50 \\
5 & 2.56 & 5.40 & \\
6 & 6.57 & 6.63 & \\
7 & & & \\
\hline
\end{tabular}


differences among the Risk Groups in their overall engagement in play activity. The Risk Groups significantly differed in their overall incidence of Quantitative Toy Play, $F(2,47)=3.31, p<.05$, as well as Exploratory, or Qualitative Toy-Play activity, $F(2,47)=3.58, p<.05$. Scheffe contrasts (at $95 \%$ confidence interval) indicated that the HR group displayed a significantly lower incidence of intervals involving quantitative and qualitative toy play. Thus, regardless of the particular levels of stress engendered by the Strange Situation episodes, the HR group appeared the least able to actively engage in play with toys.

Affect and Toy-Play Measures: Episode Interactions. There were significant episode-by-risk group interactions for the facial and vocal measures of Peak Distress Intensity: Facial $=F(12,294)=2.35$, Vocal $=F(12,294)=2.48$, both $p<.01$. Scheffe contrasts indicated that the HR group demonstrated the greatest Peak Distress Intensity, although results from facial and vocal modalities differed slightly. The HR infants showed significantly greater intensity in their facial expressions of distress than either MR or LR infants during the first separation (Episode 3). The Vocal Intensity measure for the HR infants differed to a significant extent only from that of the LR group during the first separation and subsequent reunion (Episodes 3 and 4). During the second separation and subsequent reunions all 3 Risk Groups showed increased levels of Peak Distress Intensity, thus reducing differences between the groups. To illustrate these findings, the group means of the Peak Distress summary measures for facial and vocal expression, respectively, are plotted for each episode in Figures 1 and 2.

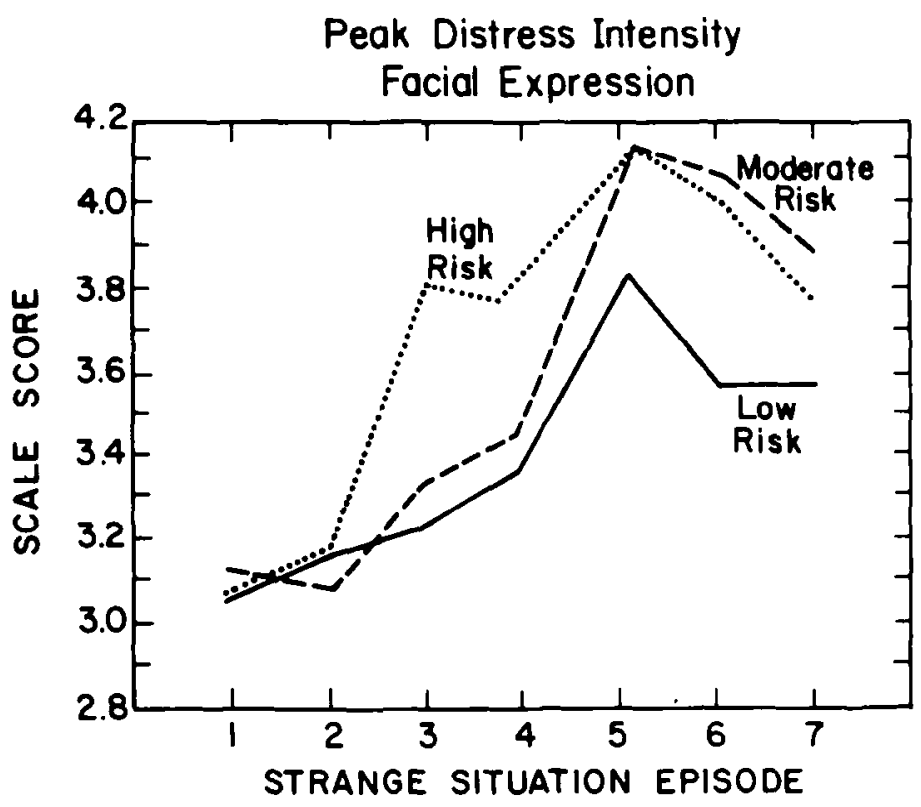

Flgure 1. Peak Distress Intensity: Facial Expression. 


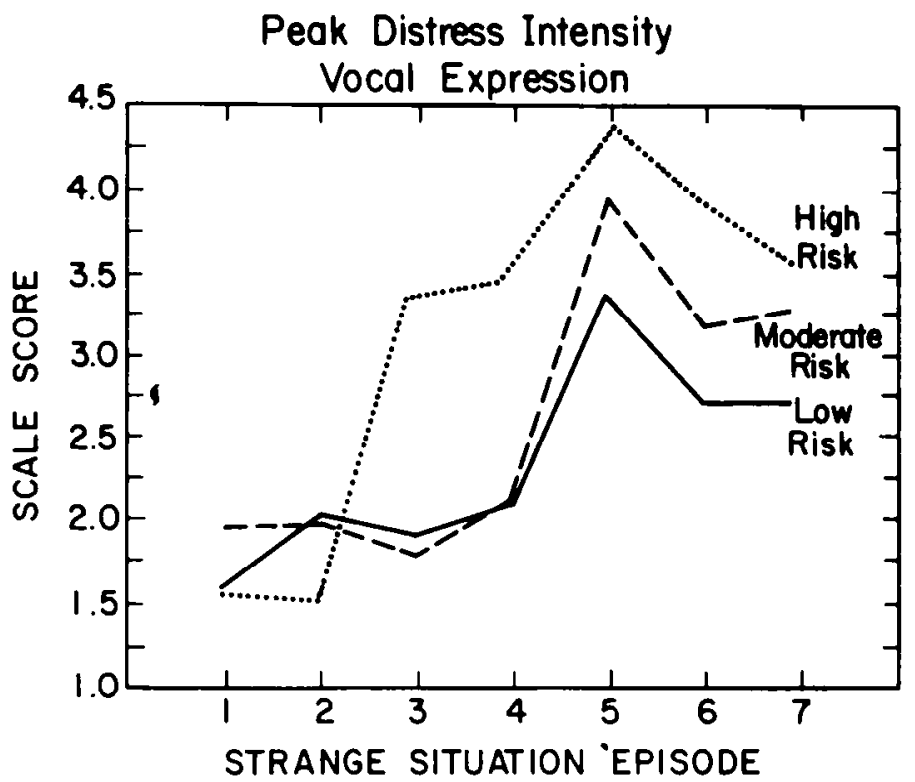

Figure 2. Peak Distress Intensity: Vocal Expression.

The Peak Distress Intensity findings can also be understood in terms of the proportion of infants in each Risk Group who were aroused to distress expression. Table 3 presents the contingency table analyses in which facial and vocal Incidence of Distress during the separation episodes (i.e., 3 and 5) are compared with risk status. Chi-square analyses demonstrate a significant associa-

TABLE 3

Facial and Vocal Indications of Distress During Separations: Number and Percentage of Subjects Demonstrating Distress by Risk Group

\begin{tabular}{|c|c|c|c|c|c|c|c|c|}
\hline & \multicolumn{6}{|c|}{ Risk Group } & \multirow[b]{3}{*}{$x^{2}$} & \multirow[b]{3}{*}{$p=$} \\
\hline & \multicolumn{2}{|c|}{$\begin{array}{l}\text { High } \\
(N=16)\end{array}$} & \multicolumn{2}{|c|}{$\begin{array}{c}\text { Moderate } \\
(N=17)\end{array}$} & \multicolumn{2}{|c|}{$\begin{array}{c}\text { Low } \\
(N=22)\end{array}$} & & \\
\hline & Absent & Present & Absent & Present & Absent & Present & & \\
\hline \multicolumn{9}{|l|}{ Facial Distress } \\
\hline Episode 4 & $\begin{array}{c}6 \\
(38 \%)\end{array}$ & $\begin{array}{c}10 \\
(62 \%)\end{array}$ & $\begin{array}{c}12 \\
(75 \%)\end{array}$ & $\begin{array}{c}4 \\
(25 \%)\end{array}$ & $\begin{array}{c}18 \\
(78 \%)\end{array}$ & $\begin{array}{c}5 \\
(22 \%)\end{array}$ & $7.843^{*}$ & .02 \\
\hline Episode 6 & $\begin{array}{c}2 \\
(13 \%)\end{array}$ & $\begin{array}{c}14 \\
(87 \%)\end{array}$ & $\begin{array}{c}3 \\
(19 \%)\end{array}$ & $\begin{array}{c}13 \\
(81 \%)\end{array}$ & $\begin{array}{c}6 \\
(26 \%)\end{array}$ & $\begin{array}{c}17 \\
(74 \%)\end{array}$ & $1.111^{*}$ & n.s. \\
\hline \multicolumn{9}{|l|}{ Vocal Distress } \\
\hline Episode 4 & $\begin{array}{c}5 \\
(31 \%)\end{array}$ & $\begin{array}{c}11 \\
(69 \%)\end{array}$ & $\begin{array}{c}13 \\
(81 \%)\end{array}$ & $\begin{array}{c}3 \\
(19 \%)\end{array}$ & $\begin{array}{c}17 \\
(74 \%)\end{array}$ & $\begin{array}{c}6 \\
(26 \%)\end{array}$ & $10.383^{*}$ & .005 \\
\hline Episode 6 & $\begin{array}{c}2 \\
(13 \%)\end{array}$ & $\begin{array}{c}14 \\
(87 \%)\end{array}$ & $\begin{array}{c}4 \\
(25 \%)\end{array}$ & $\begin{array}{c}12 \\
(75 \%)\end{array}$ & $\begin{array}{c}6 \\
(26 \%)\end{array}$ & $\begin{array}{c}17 \\
(74 \%)\end{array}$ & $1.115^{\star}$ & n.s. \\
\hline
\end{tabular}

$\star \mathrm{df}=.2$ 
tion between risk status and the presence of distress during episode 3 for both facial, $\chi^{2}(2)=7.843, p=.02$, and vocal $\chi^{2}(2)=10.383, p=.005$. The profiles of MR and LR infants are similar, indicating that approximately $20-30 \%$ of the infants in these groups demonstrated distress during the initial separaton, compared to approximately $60-70 \%$ of the infants in the HR group.

These findings concerning distress intensity indicate the HR infants displayed a greater sensitivity to distress arousal during the first separation. At higher levels of stress, as, for example, in the second separation, there is more similarity across risk groups on the measures of distress arousal and intensity of expression. However, the responses of the HR group indicated a lower threshold for distress arousal under the more moderate stress condition.

There were no significant episode-by-risk group differences for the facial and vocal Affective Range variables. This indicated that within each episode the risk groups showed a relatively similar range of distress expression despite the differences noted in Peak Distress Intensity.

Finally, there were no significant episode-by-Risk Group differences for either of the Qualitative or Quantitative Toy-Play measures. Thus, while general levels of play activity differed among the risk groups, these levels did not appear to have been differentially influenced by the events of the specific episodes.

\section{Affective and Toy-Play Temporal Measures: Main and Episode Interaction} Effects. During the second separation there were no significant Risk Group main effects for the 4 Affect temporal measures of Distress Latency, Rise Time, Initial Distress Recovery, or Final Distress Recovery. This was the case for both facial and vocal expression.' However, analysis of Initial Distress Recovery data showed significant episode-by-Risk Group interactions in both facial, $F(2,49)=4.93, p=.01$, and vocal, $F(2,49)=8.71, p=.001$, expressive modes. Scheffe contrasts indicated that the significant interaction during the 2 reunion episodes following the second separation was attributable to differences in infant recovery only during the reunion with the stranger (Episode 6). In this episode the HR infants required a significantly longer proportion of 15 -s time intervals until Initial Recovery (a distress-free interval) compared with infants in the LR group. Comparisons between the HR and MR, and MR and LR groups were not significant. Since the order of reunions with stranger and parent did not vary (i.e, the reunion with the stranger always preceded the reunion with the parent), it is impossible to interpret whether the reported differences reflect less soothing specifically in response to the efforts of a strange

\footnotetext{
'The group means for temporal measures of distress onset and recovery were influenced by the proportion of infants actually demonstrating distress. This followed from a scoring convention for the affect expression measures in which maximal scores were assigned to those infants who did not demonstrate distress. Because the proportions of infants indicating distress are, thus, confounded during the first separation, analyses of the temporal measures of affect expression were performed for only the second separation and the two subsequent reunion episodes.
} 
adult or simply a more prolonged recovery period. In either case, these findings suggest important differences between the HR infants and the LR infants in the manner which they regulate arousal.

Affective Play Recovery represents an alterantive way of describing the overall quality of infant activity during reunions. A repeated measures analysis of variance indicated a significant main effect for Risk Group, $F(2,49)=3.41$, $p<.05$, and significant episode-by-Risk interaction, $F(4,98) 2.47, p<.05$ ). Scheffe contrasts demonstrated that differences between the Risk Groups were attributable to significantly longer lags during reunions before the HR infants reengaged in toy play compared with infants in either the MR or LR groups.

\section{DISCUSSION}

The results of this study lend support to the conclusion that when placed under stress, HR preterm infants in their second year of life become more distressed and demonstrate less ability to recover from this distress and effectively reengage their environment than LR preterm infants. Of the 32 primary analyses that were performed, $25 \%$ were significant; the findings are both internally consistent and congruent with previous related studies. Thus, although the study stands in need of replication, its results are highly suggestive.

Differences are most marked when the HR and LR infants are compared. The HR infants express more distress in both the facial and vocal modalities when stress is first introduced (i.e., during the first separation episode), display less facial and vocal recovery after the second separation, and are less able to return to toy play during the reunions than the LR infants. A similar, though somewhat weaker pattern is observed when the HR and MR infants are compared. HR infants express more facial distress during the first separation and display less toy play recovery during the reunions than the MR infants. It is important to note that this pattern of reactivity is unique to the HR infants; there are no instances of MR/LR group differences. Thus, the HR infants display a pattern of more rapid and intense affect arousal, less capacity to modulate this arousal, and less ability to effectively engage their environment than preterm LR infants.

This pattern of group differences is not attributable to global differences in amount of distress expressed, or to differences in lability and range of affect displayed. The MR and LR infants have as broad a range, are as labile in their patterning of af fect expression, and are as capable of becoming distressed when the stress is sufficiently intense and cumulative as it is in the Strange Situation. In fact, under the initial, low stress conditions and the final, high stress conditions, there were no group differences in the intensity of affect expression. Instead, differences emerge in specific reference to the introduction of stress and the recovery from it. It is under these conditions that the vulnerability of the HR infants is clearly demonstrated: They are more quickly aroused and overwhelmed by distress, and are less able to modulate this arousal. 
This pattern is very consistent with observations made of LR and MR premature infants during early infancy. Researchers have consistently reported that during the neonatal period and first months of life preterm infants have a lower distress threshold, and have more difficulty modulating affect arousal and recovering state organization than healthy, full-term infants (Als, 1983; Crawford, 1982; Field, $1977 ; 1979 ; 1983)$. Our results raise the possibility that attenuation of arousal-modulation difficulties is increasingly less likely among more severely at-risk preterm infants who are small, medically compromised, and require extensive NICU intervention at birth. This conclusion conforms with the findings of those who have argued that the persistence of vulnerabilities associated with preterm birth will be most marked among sick, HR infants (see Kopp, 1983; Minde et al., 1983; Parmelee, 1975).

The results reported in this study also support the findings of Frodi and Thompson (1985), and Brachfeld et al. (1980). Although these studies differ from one another in that the Brachfeld et al. infants were not studied in a stressful situation, both studies report that LR preterm infants appeared less compromised in regard to affect arousal and modulation, and inability to engage the environment. Our results are consistent with their conclusions of attenuation of effects among LR preterm infants.

In contrast, the HR infants in this study showed a persistent pattern of lessadaptive affect arousal and modulation. However, the reasons for the patterns observed among HR infants cannot be conclusively established from the data in this study. Clearly, several hypotheses consistent with other research can be proposed. One such possibility concerns the neurophysiological immaturity or vulnerability of these infants. This immaturity, related to the biological insult of low birthweight, and severe and chronic respiratory illness combined with extensive, prolonged medical intervention in the NICU, has been associated with less stable state regulation and, possibly, with decreased abilities to modulate affective expression (Beckwith, 1976; Howard, Parmelee, Kopp, \& Littman, 1976; Parmelee, 1975). Another possibility concerns the transactional history of the infant and caregiver. This history of disrupted synchrony and/or overprotectiveness may have interfered with these infants' early experience of being able to effectively buffer and modulate affect arousal on their own (Crockenberg, 1981; Stern, 1977). Systematic observation and assessment of these infants during their first year of life would begin to clarify the ontogeny of the affective expressiveness described in this study.

Although the data reported in this investigation were collected during the Strange Situation no analysis concerning the association between infant affective regulation and the quality of mother-child attachment is presented. Some researchers claim that there is such an associaton (Frodi \& Thompson, 1985; Thompson \& Lamb, 1984), but they have not assessed affective regulation and attachment independently of one another since both measures are derived from the coding of the Strange Situation. Moreover, it is our contention that such an analysis only supplies redundant information. The qualitative distinc- 
tions and scoring criteria in the Ainsworth et al. (1978) classificatory system overlap with the type of quantitative data presented in our analysis of affect expression and modulation. The 2 approaches at best cross-validate each other. Further research is needed to address this question, utilizing a design in which affect regulation is assessed in a context different from the Strange Situation.

In short, this research supports the conclusion that there is a significant relationship between preterm infant risk status and features of affect expression in the second year of life. It suggests that early HR status is associated with less well-modulated and more easily distressed emotional expression and less adaptive toy play in later infancy. The potential implications of this persistence of effects remain to be investigated.

\section{REFERENCES}

Ainsworth, M.D.S., Blehar, M.C., Waters, E., \& Wall, S. (1978). Patterns of attachment. A psychological study of the Strange Situation. Hillsdale, NJ: Erlbaum.

Als, H. (1983). Towards a synactive theory of development: Promise for the assessment and support of infant individuality. Infant Mental Health Journal, 3, 229-243.

Als, H., Lester, B.M., Tronick, E., \& Brazelton, T.B. (1982). Towards a research instrument for the Assessment of Preterm Infants' Behavior (A.P.I.B.). In H.E. Fitzgerald, B.M. Lester, \& M.W. Yogman (Eds.), Theory and research in behavioral pediatrics (Vol. 1). New York: Plenum.

Bakeman, R., \& Brown, J.V. (1980). Early interaction: Consequences for social and mental development at three years. Child Development, 51, 437-447.

Ballard, J.L., Novak, K.K., \& Driver, M. (1979). A simplified score for assessment of fetal malnutrition in newly born infants. Journal of Pediatrics, 95, 769-774.

Beckwith, L. (1976). Caregiver-infant interaction as a focus for therapeutic intervention with human infants. In R.N. Walsh \& W.T. Greenough (Eds.), Environments as therapy for brain dysfunction. New York: Plenum.

Brachfeld, S., Goldberg, S., \& Sloman, J. (1980). Parent-infant interaction in free play at 8 and 12 months: Effects of prematurity and immaturity. Infant Behavior and Development, 3 , 289-305.

Cohen, J. (1960). A coefficient of agreement for nominal scales. Educational and Psychological Measurement, 20, 37-46.

Cohen, J. (1968). Weighted kappa: Nominal scale agreement with provision for scaled disagreement or partial credit. Psychological Bulletin, 70, 213-219.

Crawford, J.W. (1982). Mother-infant interaction in premature and full-term infants. Child Development, 53, 957-962.

Crnic, K.A., Ragozin, A.S., Greenberg, M.T., Robinson, N.M., \& Basham, R.B. (1983). Social interaction and developmental competence of preterm and full-term infants during the first year of life. Child Development, 54, 1199-1210.

Crockenberg, S. (1981). Infant irritability, mother responsiveness, and social support influences on the security of infant-mother attachment. Child Development, 52, 857-865.

DiVitto, B., \& Goldberg, S. (1979). The effects of newborn medical status on early parent-infant interaction. In T.M. Field, A.M. Sostek, S. Goldberg, \& H.H. Shuman (Eds.), Infants born at risk: Behavior and development. New York: Spectrum.

Duffy, F.H., Mowrer, G., Jensen, F., \& Als, H. (1984). Neural plasticity: A new frontier for infant development. In H.E. Fitzgerald, B.M. Lester, \& M.W. Yogman (Eds.), Theory and research in behavioral pediatrics (Vol. 2). New York: Plenum. 
Emde, R., Gaensbauer, T., \& Harmon, R. (1976). Emotional expression in infancy: A biobehavioral study. Psychological Issues Monograph Series, 10 (Monograph No. 37).

Field, T.M. (1977). Maternal stimulation during infant feeding. Developmental Psychology, 13, $539-540$.

Field, T., (1979). Games parents play with normal and high-risk infants. Child Psychiatry and Human Development, 10, 41-48.

Field, T. (1982). Affective displays of high-risk infants during early interactions. In T. M. Field \& A. Fogel (Eds.), Emotion and early interaction. Hillsdale, NJ: Erlbaum.

Field, T.M. (1983). Early interactions and interaction coaching of high-risk infants and parents. In M. Perlmutter (Ed.), The Minnesota Symposia on Child Psychology (Vol. 16). Hillsdale, NJ: Erlbaum.

Field, T.M., \& Fogel, A. (Eds.).(1982). Emotion and early interaction. Hillsdale, NJ: Erlbaum.

Frodi, A., \& Thompson, R. (1985). Infants' affective responses in the Strange Situation: Effects of prematurity and of quality of attachment. Child Development, 56, 1280-1290.

Gaensbauer, T.J., \& Harmon, R.J. (1981). Clinical assessment in infancy utilizing structured playroom situations. Journal of the American Academy of Child Psychiatry, 20, 264-280.

Greene, J.G., Fox, N.A., \& Lewis, M. (1983). The relationship between neonatal characteristics and three-month mother-infant interaction in high risk infants. Child Development, 54, 1286-1296.

Howard, J., Parmelee, A., Kopp, C., \& Littman, B. (1976). A neurological comparison of preterm and full-term infants at term conceptional age. Journal of Pediatrics, 88, 995-1002.

Kopp, C.B. (1983). Risk factors in development. In M.M. Haith \& J.J. Campos (Eds.), Infancy and developmental psychobiology. New York: Wiley.

Lewis, M., \& Michalson, L. (1983). Children's emotions and moods: Developmental theory and measurement. New York: Plenum.

Minde, K., Whitelaw, A., Brown, J., \& Fitzhardinge, P. (1983). Effect of neonatal complications in premature infants on early parent-child interactions. Developmental Medicine \& Child Neurology, 25, 763-777.

Parmelee, A.H. (1975). Neurophysiological and behavioral organization of premature infants in the first months of life. Biological Psychiatry, 10, 473-483.

Stern, D. (1977). The first relationship: Infant and mother. Cambridge, MA: Harvard University Press.

Thompson, R.A. (1981). Continuity and change in socioemotional development during the second year. Unpublished doctoral dissertation. The University of Michigan: Ann Arbor.

Thompson, R.A., \& Lamb, M.E. (1984). Assessing qualitative dimensions of emotional responsiveness in infants: Separation reactions in the Strange Situation. Infant Behavior and Development, 7, 423-445. 